\title{
An Efficient Routing Tree Algorithm for Combined Uplink and Downlink Subframes on Centralized Scheduling in IEEE 802.16 Based Wireless Mesh Networks
}

\author{
Ali Behfarnia, Vahid Tabataba Vakili \\ Department of Electrical Engineering, Iran University of Science and Technology, Tehran, Iran \\ E-mail: Behfarnia@elec.iust.ac.ir, Vakily@iust.ac.ir \\ Received January 24, 2011; revised March 18, 2011; accepted March 20, 2011
}

\begin{abstract}
IEEE 802.16 mesh mode defines routing tree for transmitting data in centralized scheduling but it does not define any explicit proposal for combining uplink and downlink subframes. Deploying combined uplink and downlink subframes on the centralized scheduling scheme can be more flexible and utilization is improved. However, existing interferences among the transmission of neighboring nodes lead to performance reduction of the network. In this paper, an efficient routing tree algorithm is proposed with combined uplink and downlink slot allocation on the centralized scheduling scheme which can abate interferences in the network. This algorithm allows more subscriber stations to transmit concurrently and so improves spatial reuse in the network. Also, the algorithm uses multi-channel and single channel systems and considers relay model, smoothing switching frequently between transmitting and receiving in successive time slots and fairness in the network. Extensive simulation results demonstrate the effectiveness of the proposed method in terms of scheduling length, link concurrency ratio, network throughput and Channel Utilization Ratio (CUR).
\end{abstract}

Keywords: IEEE 802.16, Wireless Mesh Network, Centralized Scheduling, Slot Allocation, Routing Tree

\section{Introduction}

Wireless mesh network (WMN) is one of the attractive technologies to achieve better services in next generation wireless networks. The WMNs can be dynamically self-configured and self-organized, with the nodes within the mesh network automatically establishing and maintaining mesh connectivity among themselves. These features lead to advantages such as easy network maintenance, robustness, low up-front cost and reliable service coverage [1]. Also, the WMNs can be integrated with other networks such as IEEE 802.11, IEEE 802.16, ad hoc networks, cellular networks, etc.

Medium access control (MAC) layer in the IEEE 802.16 protocol stack supports two operation modes: PMP (point-to-multi-point) and mesh (multipoint-tomultipoint) [2]. All subscriber stations (SSs) in the PMP mode are managed by base station (BS) and each SS has to communicate with the BS before transmitting data to other SSs. In contrast, all mesh SSs (MSSs) within the network usually communicate with each other without coordinating with the mesh BS (MBS).
IEEE 802.16 mesh mode employs time division multiple access (TDMA) as the channel access method, where each radio channel includes multiple frames. A frame can be divided into time slots which can be assigned to each SS and the BS. There are two scheduling schemes for allocating these time slots to all SSs within the mesh network. One of them is centralized scheduling and the other is distributed scheduling. In the centralized scheme, all traffic is to or from the BS and so this scheme is preferable for transmitting internet traffic. All traffic will be transmitted between SSs in the distributed scheduling scheme and so this scheme is preferable for transmitting intranet traffic. In both centralized and distributed schemes, spatial reuse can be exploited for improvement of wireless resource and network throughput. The spatial reuse in TDMA means that multiple links or nodes can transmit in a time slot simultaneously only if the transmissions do not lead to collision. In what fallows, we will focus on the centralized scheduling in IEEE 802.16 mesh mode.

Several works have been tried to improve network throughput and spatial reuse. Han in [3,4] proposed scheduling algorithm for uplink traffic by using relay 
model and constructing routing tree in centralized mode but did not consider downlink traffic and multi-channel system. Liu in [5] designed uniform slot allocation algorithms which exploit combined uplink and downlink slot allocation based on the centralized scheduling but did not consider an efficient routing algorithm and multi-channel system. Authors in [6,7] proposed centralized scheduling and channel assignment for IEEE 802.16 mesh networks but did not focus on downlink traffic and efficient routing algorithm. Sheu in [8] proposed an interferenceaware routing tree algorithm for the centralized scheduling but did not consider combined uplink and downlink traffic of each node and multi-channel system. Al- Hemyari in $[9,10]$ proposed a cross-layer design between network layer and MAC layer but did not discuss about the downlink parameters and combined uplink and downlink slot allocation.

In this paper, an efficient and interference-aware routing tree algorithm is proposed which exploits combined uplink and downlink slot allocation on the centralized scheduling. The proposed method considers interferences, downlink packets, uplink packets and node identifier for determining parent nodes in routing tree construction. This algorithm can improve spatial reuse and network throughput. Multi-channel system is used to abate interference from transmission of all neighboring nodes within the mesh network. In addition, this scheduling algorithm considers fairness among the nodes within the network and moderates switching frequently between transmitting and receiving in successive time slots. This algorithm is evaluated through some important metrics such as scheduling length, channel utilization ratio, network throughput and link concurrency ratio. To the best of authors' knowledge, no research work has been proposed to address the routing tree method with considering uplink and downlink traffic in combined uplink and downlink slot allocation on the centralized scheduling.

The rest of this paper is organized as follows. In section 2, we will introduce the system model in IEEE 802.16 mesh mode. In section 3, we will explain the proposed algorithm in detail. Section 4 shows the simulation results. Finally, the paper is concluded in section 5.

\section{Basic Scheduling Scheme in IEEE 802.16 Mesh Mode}

In this section, overview on frame structure of the IEEE 802.16 mesh mode and routing tree are introduced.

\subsection{Frame Structure in IEEE 802.16 Mesh Mode}

As mentioned above, each radio channel can be divided into frames. A frame consists of control subframe and data subframe, as shown in Figure 1. Each control subframe is further divided into network control subframe and schedule control subframe. The network control subframe creates and maintains the network by mesh network entry (MSH-NENT) message and mesh network configuration (MSH-NCFG) message. These messages are transmitted periodically in the mesh network. If the frame has not network control subframe, it has schedule control subframe. The schedule control subframe consists of centralized scheduling and distributed scheduling for sharing nodes within the network in a common radio resource. In centralized scheduling scheme, all SSs send their recourse requests to the $\mathrm{BS}$ by mesh centralized scheduling (MSH-CSCH) message. The BS receives these resource requests and determines flow assignments of all SSs. The SSs are informed about these assignments through MSH-CSCH. Another message is called mesh centralized scheduling configuration (MSH-CSCF). This message is broadcasted by the BS to disseminate information about routing tree and whole centralized scheduling configuration among all SSs within the mesh network. The distributed scheduling scheme can be classified into coordinated and uncoordinated. Each SS in the coordinated distributed scheduling must coordinate itself by its two hops neighbor for a collision free data transmission. But, schedules are determined by directed requests and grants between each two SS in uncoordinated distributed (like ad-hoc networks). Both coordinated and uncoordinated schemes employ mesh distributed scheduling (MSH-DSCH) message to convey resource requests and grants to the neighbors [2].

\subsection{Routing Tree}

To construct the routing tree, if a new station wants to join the mesh network, it must scan the MSH-NCFG message to synchronize with the network. Then, the new station selects a parent node among all possible neighbors according to specific policy (which will be discussed in next section). After that, the new station transmits a MSH-NENT message with registration information to the MBS via its parent node. After receiving the registration message of new station, the MBS adds the new station to the network as a child of determined parent

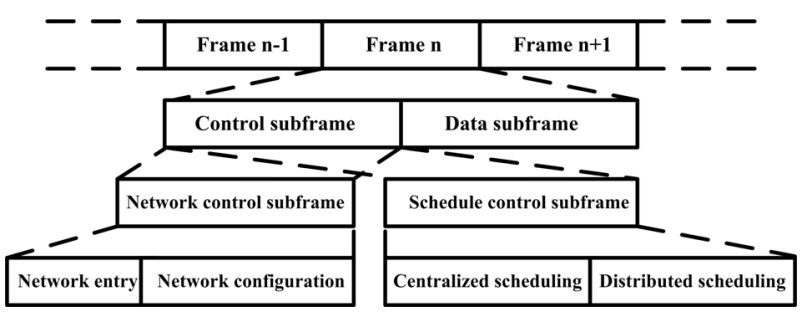

Figure 1. Frame structure for IEEE 802.16 based WMNs. 
node.

In the routing tree construction procedure, the hopcount of the MBS is assumed to be zero and all neighbors of MBS are assigned to be one. A station selects its parent node based on smallest hop-count and then hop-count of it is assigned to be its parent node plus one. Figure 2(a) gives an example of mesh topology and routing tree, where the dashed lines show the mesh topology and the solid lines represent the routing tree.

\section{Proposed Algorithm}

\subsection{Motivation and Problem Overview}

Several issues can be considered concerning the centralized scheduling scheme. The issues can be categorized in three parts:

- Routing tree: The policy of routing tree construction influences on system performance.

- Resource management: resource requests from all SSs must be managed by the BS. Also, the BS must determine the uplink and downlink flow assignments in such a way that overload does not occur in the network.

- Scheduling algorithm: all SSs should be scheduled without interferences to achieve higher spatial reuse and network throughput in the mesh network.

In this work, we will focus on the routing tree and scheduling algorithm. Resource management is assumed to be known in the proposed method. In the other words, we first design a routing algorithm and then schedule the minislots of data subframe according to the routing tree.

This work can be called the routing and packet scheduling (RPS), which has become an important issue in the centralized scheduling. To illustrate the issue more clearly, let us consider the example of routing tree in Figure 2(a). A single channel time division duplex model is assumed to be adopted in this network and each SS is assumed has only one packet for uplink transmission and one packet for downlink transmission (homogeneous). Figures 2(b)-(c) represents the scheduling matrix (SM) which is based on routing tree shown in Figure 2(a). The terms $1 \downarrow$ and $1 \bullet$ which are shown in the SM are used for transmitting downlink and uplink traffic, respectively. Among several criteria to select the nodes for sending packets, we select the least hop-count to the BS for uplink transmissions and largest hop-count from the BS for downlink transmission, because these selections are demonstrated to be the best in [4,5]. Also, we can find from the [5] that for combined uplink and downlink slot allocation (CSLA), the downlink slot allocation is implemented first and then followed by uplink slot allocation. Thus, it is adopted in our example. As we see from the Figures 2(b)-(c), the node SS10 which with the largest hop-count from the BS and largest identifier is transmitted first by the BS. In this condition, nodes SS1and SS3 cannot transmit and receive. Also SS4 and SS6 cannot transmit with considering interference model. The SS8 can transmit its uplink traffic to SS4 without interference at the first time slot. If all nodes that are not interfered with each other have ended their transmissions at a time slot, the scheduling of that time slot is finished. Thus,

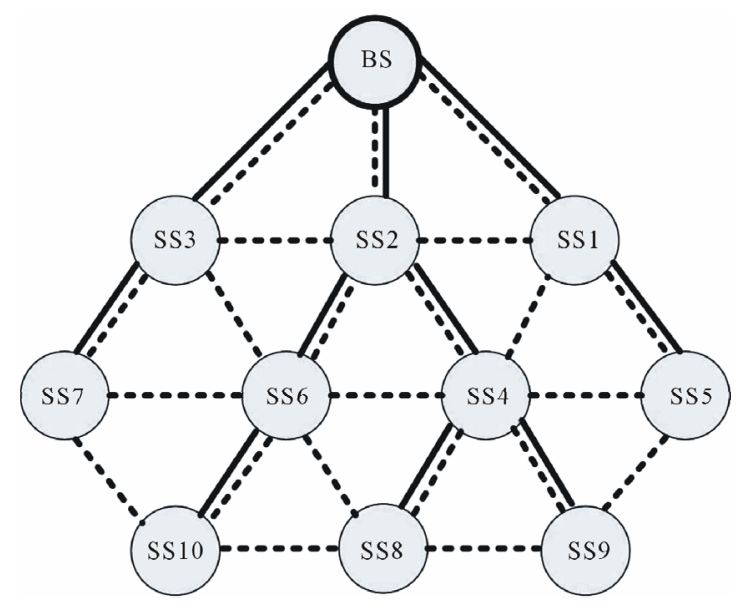

(a)

\begin{tabular}{|c|c|c|c|c|c|c|c|c|c|c|c|c|c|c|c|}
\hline $\mathrm{k}$ & 1 & 2 & 3 & 4 & 5 & 6 & 7 & 8 & 9 & 10 & 11 & 12 & 13 & 14 & 15 \\
\hline $\mathrm{BS}$ & 10 & 0 & $5 \bullet$ & 9 & 0 & 7 & 8 & 0 & 3 & 6 & 0 & 4 & 0 & 2 & 1 \\
\hline SS 1 & 0 & 0 & 0 & 0 & 0 & 0 & 0 & 0 & 0 & 0 & 5 & 0 & 0 & 0 & 0 \\
\hline SS2 & 0 & 10 & 0 & 0 & 9 & 0 & 0 & 8 & 0 & 0 & 6 & 0 & $4 \bullet$ & 0 & 0 \\
\hline SS3 & 0 & 0 & 0 & 0 & 0 & 0 & 0 & 7 & 0 & 0 & 0 & 0 & 0 & 0 & 0 \\
\hline SS4 & 0 & 0 & 0 & 0 & 0 & 9 & 0 & 0 & 8 & 0 & 0 & 0 & 0 & 0 & 0 \\
\hline SS5 & 0 & 0 & 0 & 0 & 0 & 0 & 0 & 0 & 0 & 0 & 0 & 0 & 0 & 0 & 0 \\
\hline SS6 & 0 & 0 & 10 & 0 & 0 & 0 & 0 & 0 & 0 & 0 & 0 & 0 & 0 & 0 & 0 \\
\hline SS7 & 0 & 0 & 0 & 0 & 0 & 0 & 0 & 0 & 0 & 0 & 0 & 0 & 0 & 0 & 0 \\
\hline SS8 & 8 & 0 & 0 & 0 & 0 & 0 & 0 & 0 & 0 & 0 & 0 & 0 & 0 & 0 & 0 \\
\hline SS9 & 0 & 0 & 0 & $9 \bullet$ & 0 & 0 & 0 & 0 & 0 & 0 & 0 & 0 & 0 & 0 & 0 \\
\hline SS10 & 0 & 0 & 0 & 10 & 0 & 0 & 0 & 0 & 0 & 0 & 0 & 0 & 0 & 0 & 0 \\
\hline
\end{tabular}

(b)

\begin{tabular}{|c|c|c|c|c|c|c|c|c|c|c|c|c|c|c|c|}
\hline $\mathrm{k}$ & 16 & 17 & 18 & 19 & 20 & 21 & 22 & 23 & 24 & 25 & 26 & 27 & 28 & 29 & 30 \\
\hline $\mathrm{BS}$ & 0 & 0 & 0 & 0 & 0 & 0 & 0 & 0 & 0 & 0 & 0 & 0 & 0 & 0 & 0 \\
\hline $\mathrm{SS} 1$ & $1 \bullet$ & 0 & 0 & 0 & 0 & $5 \bullet$ & 0 & 0 & 0 & 0 & 0 & 0 & 0 & 0 & 0 \\
\hline SS2 & 0 & $2 \bullet$ & 0 & 0 & $4 \bullet$ & 0 & 0 & $6 \bullet$ & 0 & 0 & $8 \bullet$ & 0 & $9 \bullet$ & 0 & $10 \bullet$ \\
\hline SS3 & 0 & 0 & $3 \bullet$ & 0 & 0 & 0 & 0 & 0 & $7 \bullet$ & 0 & 0 & 0 & 0 & 0 & 0 \\
\hline SS4 & 0 & 0 & 0 & $4 \bullet$ & 0 & 0 & 0 & 0 & 0 & $8 \bullet$ & 0 & $9 \bullet$ & 0 & 0 & 0 \\
\hline SS5 & 0 & 0 & $5 \bullet$ & 0 & 0 & 0 & 0 & 0 & 0 & 0 & 0 & 0 & 0 & 0 & 0 \\
\hline SS6 & 0 & 0 & 0 & 0 & 0 & 0 & $6 \bullet$ & 0 & 0 & 0 & 0 & 0 & 0 & $10 \bullet$ & 0 \\
\hline SS7 & $7 \bullet$ & 0 & 0 & 0 & 0 & 0 & 0 & 0 & 0 & 0 & 0 & 0 & 0 & 0 & 0 \\
\hline SS8 & 0 & 0 & 0 & 0 & 0 & 0 & 0 & 0 & 0 & 0 & 0 & 0 & 0 & 0 & 0 \\
\hline SS9 & 0 & 0 & 0 & 0 & 0 & 0 & 0 & 0 & 0 & 0 & 0 & 0 & 0 & 0 & 0 \\
\hline SS10 & 0 & 0 & 0 & 0 & 0 & 0 & 0 & 0 & 0 & 0 & 0 & 0 & 0 & 0 & 0 \\
\hline
\end{tabular}

(c)

Figure 2. (a) Example of mesh topology and routing tree, (b)-(c) scheduling matrix based on the routing tree in (a). 
scheduling the first time slot is finished. In the next time slot, node SS2 forwards the downlink traffic of SS10 to the SS6. In this condition, with considering the interference model, nodes SS1, SS3 and SS4 cannot receive the traffic from other nodes and SS3, SS4, SS7, SS8 and SS10 cannot transmit to other nodes. Thus only link SS2SS6 is scheduled in this time slot. With the same procedure, scheduling matrix is constructed in the Figures 2(b)-(c), where 30 time slot is required to transmit the traffic of all nodes to their destinations.

Constructing efficient routing tree algorithm for allowing more nodes to transmit simultaneously at a time slot is the goal of this paper. With considering the construction of routing tree in the network layer and scheduling algorithm in the MAC layer, the cross layer concept can be formed in our mind. In the network layer, all information about routes and interferences of the nodes within the network are acquired from BS. In the MAC layer, all centralized scheduling information is sent to the routing tree algorithm in network layer. After establishing the routes of the nodes, the paths of routing tree are fed to the MAC layer. With considering the channel assignment of multi-channel system in the MAC layer and implementing centralized scheduling, we can achieve the reduction of interferences in the network.

\subsection{Interference Model and Channel Assignment}

There are two types of interferences in the wireless networks in which the nodes use a shared medium to communicate with each other. The first type is called primary interference which occurs when a station has to transmit or receive at one time. The other type is called secondary interference which occurs when a station $\mathrm{M}$ who intends to receive from station $\mathrm{N}$ is within the range of another transmitter whose transmissions, though not intended for $\mathrm{M}$, interfere with the transmissions of $\mathrm{N}$.

For the single channel single transceiver mode, the following interference model is assumed in the mesh network:

- Each node's radio is assumed half duplex, and so nodes cannot receive and transmit in one time. Also, all nodes cannot receive from multiple nodes or transmit to multiple nodes in one time.

- All nodes in the range of the transmitter except receiver cannot receive from other nodes and all nodes in the range of receiver except transmitter cannot transmit, because interference occurs in these conditions.

In respect to the use of single transceiver for each node in the network, the primary interference cannot be eliminated. However, by using multi-channel single trans- ceiver system each node can employ one channel at each time slot, and also can employ other channel in the other time slots. Thus, different stations can use different channel in a time slot and so a lot of nodes can transmit/receive simultaneously. Hence, with exploiting multi-channel system the secondary interferences can be eliminated.

For illustrating the channel assignment of the multichannel system more lucidly, we can return to the scheduling matrix and routing tree which are shown in Figure 2. According to use of the single channel system and single transceiver, in the second time slot of the scheduling matrix, only SS2 can transmit its packets to SS6. However, if the multi-channel system was used in this network with the same condition, the link BS-SS3 would be activated for downlink transmission and also link SS5-SS1 and SS9-SS4 would be activated for uplink transmission.

\subsection{Proposed Routing Tree Algorithm Common}

The goal of our routing algorithm is to find the best paths between the static SSs and the BS in the routing tree topology. In this algorithm four parameters are considered; number of hop-count from the BS, node identification (i.e. ID node), number of interfering nodes and the number of uplink and downlink traffic per each node. The proposed algorithm is implemented based on the following steps:

1) Select the parent node based on the least hop-count from the BS.

2) If there is one node in the transmission range, choose it as the parent node, else go to step 3.

3) Calculate the number of neighboring nodes that lead to interference from the selected nodes and then choose the parent nodes with the least number of interference.

4) If there is one node with the least number of interference, choose it as the parent node, else go to step.

5) Calculate the load of the routes which include the candidate parent nodes. This parameter can be obtained as summation of all uplink packets and downlink packets of the nodes along the route to the BS. After that, choose the parent node in the path with the minimum load.

6) If there is one path, choose corresponding parent node, else go to step 7.

7) Select the parent node based on minimum ID node.

The ambiguity of the routing tree construction with this algorithm can be abolished by a simple example. Let's return to mesh topology in Figure 2(a) and assume each node has one packet for uplink transmission and one packet for downlink transmission. In this condition, choosing parent nodes in accordance with the proposed 
algorithm can be implemented as follows. SS1, SS2 and SS3 have one upper layer station and so they choose it as their parent node. SS4 can select SS1 and SS2 as its parent node and so the algorithm will enter to the third step. With considering the interference number of candidate nodes, SS4 selects SS1 as its parent node. SS5 has only one upper level node and so it will select SS1 as the parent node. SS6 and SS7 select SS3 as their parent node with the similar reasons of SS4 and SS5. SS8 has SS4 and SS6 in its upper layer. These two stations have same condition in interference number and so the proposed algorithm will enter to the step 5. But, these two parent nodes have similar value in their load of routing paths which are equal to 4 ( 2 uplink packets plus 2 downlink packets). In this condition, the algorithm will enter to the step 7 and so SS8 select SS4 as its parent node. SS9 select SS5 between SS5 and SS4 as the parent node because the interference number of SS5 is lower than SS4. With the same reason SS10 chooses SS7 as its parent node. Figure 3 shows the proposed routing tree by the solid lines.

\subsection{Centralized Scheduling Scheme}

From the resource management, all SSs and the BS are assigned service token (ST) based on their uplink and downlink traffic demands. The ST which is assigned to each SS for uplink or downlink (at the BS) demands can be obtained as its traffic demands divided by greatest common divisor of all SSs traffic demands. For example, if traffic demands of SSs are $2 \mathrm{Mbps}, 4 \mathrm{Mbps}, 6 \mathrm{Mbps}$ and $10 \mathrm{Mbps}$, the STs which are assigned to SSs will be $1,2,3$ and 5, respectively. With this assignment, the fairness can be guaranteed because the nodes within the network will not be starved. Downlink service token matrix (DSTM) and uplink service token matrix (USTM) can be constructed from downlink and uplink ST demands of all nodes.

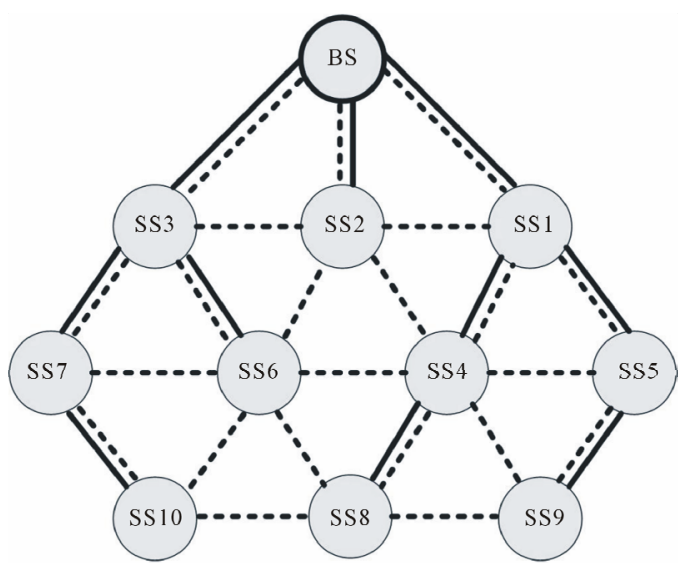

Figure 3. The proposed routing tree algorithm.
The link selection criterion for implementing the slot allocation algorithm is adapted to the CSLA method which is discussed in [5]. Therefore, we select the least hop-count to BS for uplink transmissions and largest hop-count from the BS for downlink transmission. Also, downlink slot allocation has higher priority than uplink slot allocation in each time slot. In each link selection process, transmit node $\left(T \_n\right)$, receive node $\left(R \_n\right)$ and source node $\left(S \_n\right)$ of each link are obtained. The $S \_n$ refers to the source node of ST and the $T \_n$ and $R \_n$ are relay nodes in the route between $S \_n$ and its destination.

After each slot allocation, the corresponding matrixes will be adjusted and SM will be set. Setting the SM is as follows:

$$
\mathrm{SM}\left(T \_n, k\right)=S \_n
$$

Also, adjusting the DSTM and USTM are as follow:

$$
\begin{aligned}
& \operatorname{DSTM}\left(T \_n, S \_n\right)=\operatorname{DSTM}\left(T \_n, S \_n\right)-1 \\
& \operatorname{DSTM}\left(R \_n, S \_n\right)=\operatorname{DSTM}\left(R \_n, S \_n\right)+1 \\
& \operatorname{USTM}\left(T \_n, S \_n\right)=\operatorname{USTM}\left(T \_n, S \_n\right)-1 \\
& \operatorname{USTM}\left(R \_n, S \_n\right)=\operatorname{USTM}\left(R \_n, S \_n\right)+1
\end{aligned}
$$

Relation (1) means that $T \_n$ is scheduled at time slot $k$ in the SM. The service token of corresponding $S \_n$ at the transmitter is diminished by one, and the service token of corresponding $S \_n$ at the receiver is increased by one, as in (2) and (3). Likewise, the USTM will be adjusted similar to DSTM, as in (4) and (5). In this algorithm, the first input first output (FIFO) is assumed as the buffer of each node. Also, each receiving node forwards the traffic after receiving all the traffic from its transmitter node. In the other words, each receiving node collects all ST of its transmitter and then transmits the traffic. This procedure leads to reduce switching frequently between transmitting and receiving nodes in successive time slots. For more information about switching frequently between receiving and transmitting within two adjacent time slots, we refer interested readers to [5].

Figure 4 gives the SM in accordance with the proposed routing tree algorithm which is shown in Figure 3. Figure 4(a) gives the SM with using single channel system and Figure 4(b) gives the SM with using multichannel system. In these SMs, we assume that the value of ST for each node in uplink and downlink direction is to be one (homogeneous). As we see from these figures, we can find that 23 and 20 time slots are required to convey the traffic of all nodes to their destinations which is less than traditional structure which is shown in Figure 2.

\section{Simulation}

\subsection{Performance Metrics}

The performance of the proposed algorithm is evaluated 


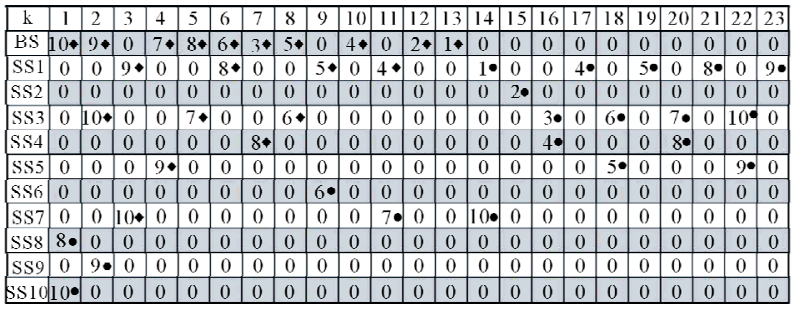

(a)

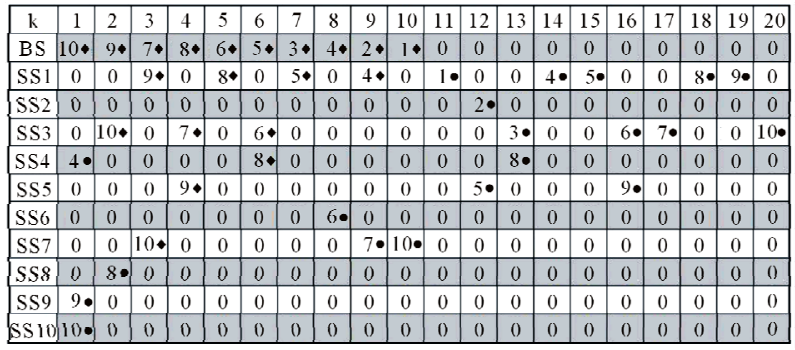

(b)

Figure 4. Scheduling matrixes of the proposed algorithm with using (a) single channel system and (b) multi-channel system.

through scheduling length, normalized network throughput, channel utilization ratio (CUR) and link concurrency ratio (LCR). The scheduling length is the number of time slots which is required to transmit the traffic of all SSs and the BS to their destinations in a cycle. The cycle of a link scheduling is the time needed to transmit all the data traffic under certain traffic model in the WMN. The scheduling length is one of the prevalent criteria in the scheduling algorithm which is considered in most of existing literature. Normalized network throughput is employed for evaluating the network performance which can be calculated as the total number of STs of all nodes divided by scheduling length. The CUR can be obtained as the number of occupied time slots in SM divided by number of all possible time slots. All possible time slots can be obtained as the scheduling length multiplied by the number of nodes. Transport capacity of the mesh network can be improved via higher CUR. The LCR is the average number of nodes that can transmit data simultaneously at a time slot. This parameter is employed for evaluating the spatial reuse efficiency in the mesh network.

\subsection{Simulation Setup}

We compare the proposed algorithm with the CSLA method in [5] which is proposed for combining uplink and downlink slot allocation in the centralized scheduling. Also, the results compare with uplink and downlink slot allocation which are executed separately.
We use $\mathrm{C}$ programming language to evaluate the performance of the proposed algorithm. A square area of size 50 by 50 units is used for distributing all SSs in the mesh network randomly and uniformly. The BS is located on the center of the square area. A same transmission range is assumed for all SSs and the BS. Two stations will be neighbors if the distance between them is smaller than their transmission range. The transmission range of each node is assumed to be 10 units. The service token number of the nodes is randomly selected between 0 and 5 (heterogeneous) and 1 (homogeneous). Simulations are implemented for the range of nodes from 20 to 100 with increment step of 10 . Results presented in this section are averaged among 500 connected graphs.

\subsection{Simulation Results}

Figures 5-8 gives the scheduling length, channel utilization ratio, link concurrency ratio and normalized network throughput. The meaning of proposed-sig and proposed-mul that are used in these figures refer to use of single channel and multi-channel systems with the proposed algorithm.

As we see from the Figure 5, the scheduling length increases with the number of nodes because with increasing the nodes in the network, the traffic demand is also increased. Moreover, we can find that with use of multi-channel system the scheduling length is significantly improve, and this is because with employing multi-channel system more subscriber stations can transmit simultaneously.

From the Figure 6, we can observe that increasing the number of nodes lead to decrease the CUR because with increasing the nodes, interfering nodes is also increased. Also, with exploiting multi-channel system in the algorithm the CUR can significantly improve in compare to use of single channel system. The reason is due to use of same channel at the same time slots which lead to increment the interferences which are sorely affected on simultaneous transmission. Moreover, we can find that the CUR is usually below $10 \%$ for the number of nodes more than 20 because the nodes which are at the first hop-count from the BS act as bottlenecks in compare to the nodes with higher number of hop-count which idle in most of the time.

Figure 7 presents the simulation results of the link concurrency ratio. We notice that the LCR in the proposed algorithm using multi-channel system does not ascendant in this figure. This fact stems from the primary interference. In the other words, with using multi-channel system and increasing the nodes in the network, the primary interference occurred more than previously in the network. 


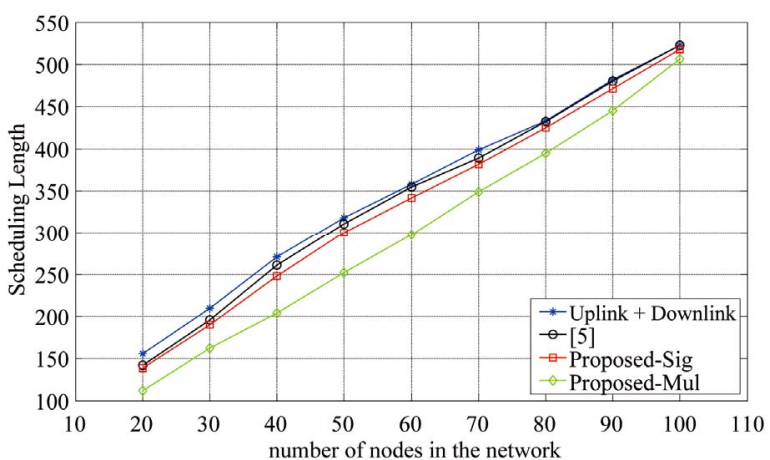

(a)

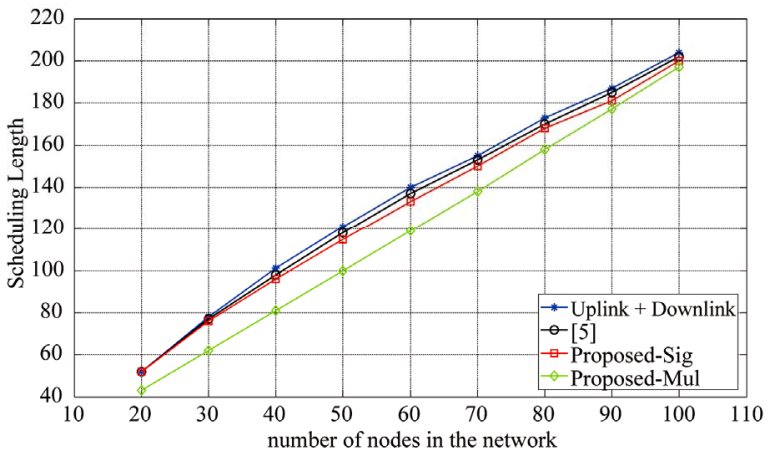

(b)

Figure 5. Scheduling length. (a) Service token of all nodes $\in[0,5]$; (b) Service token of all nodes $=\mathbf{1}$.

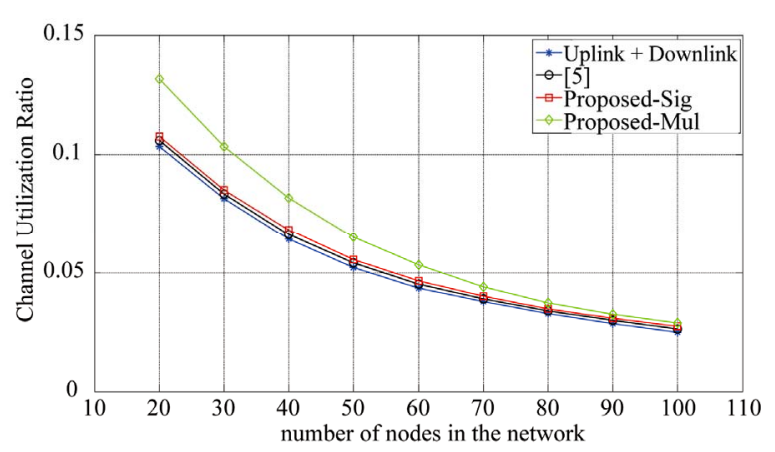

(a)

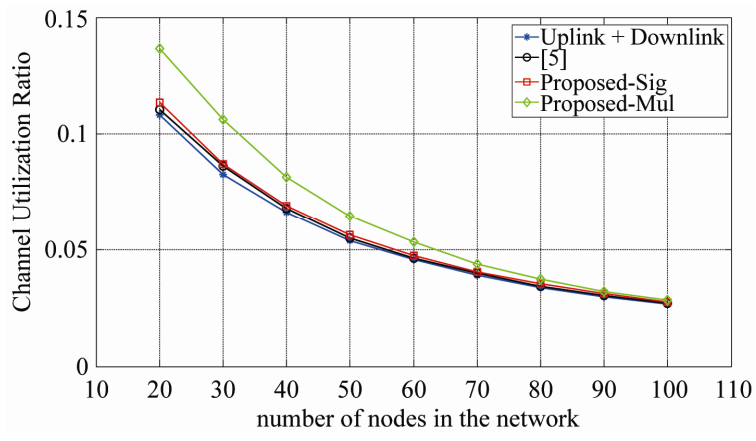

(b)

Figure 6. Channel utilization ratio. (a) Service token of all nodes $\in[0,5]$; (b) Service token of all nodes $=\mathbf{1}$.

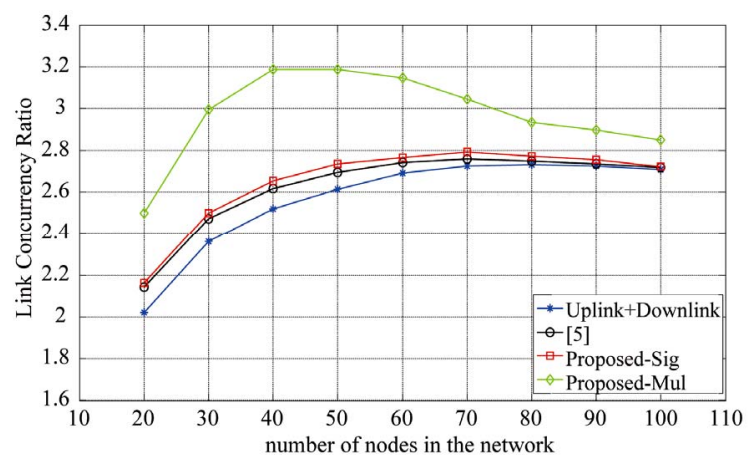

(a)

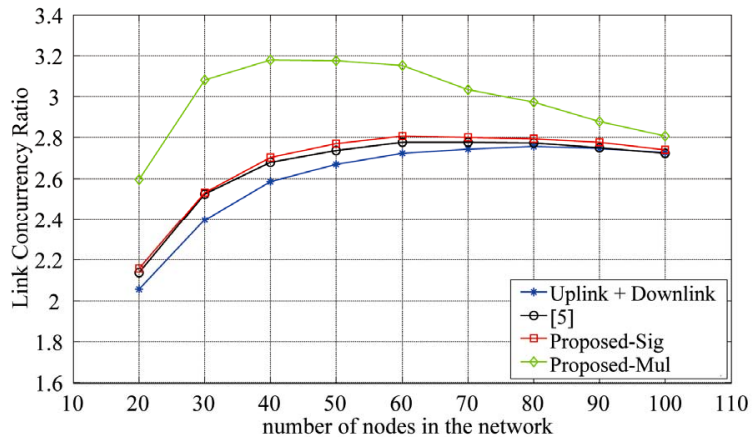

(b)

Figure 7. Link concurrency ratio. (a) Service token of all nodes $\in[0,5]$; (b) Service token of all nodes $=\mathbf{1}$.

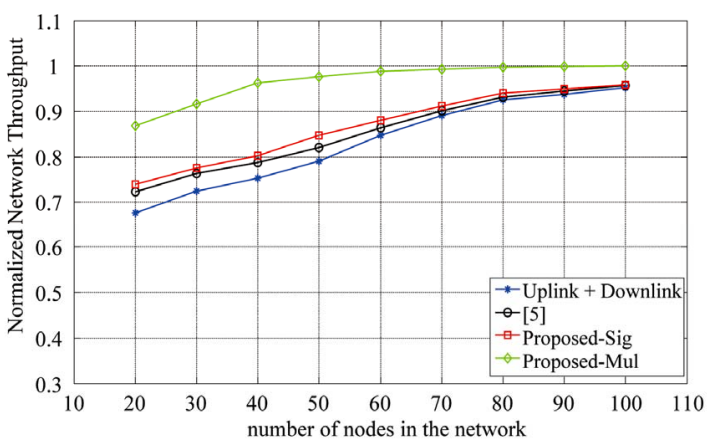

(a)

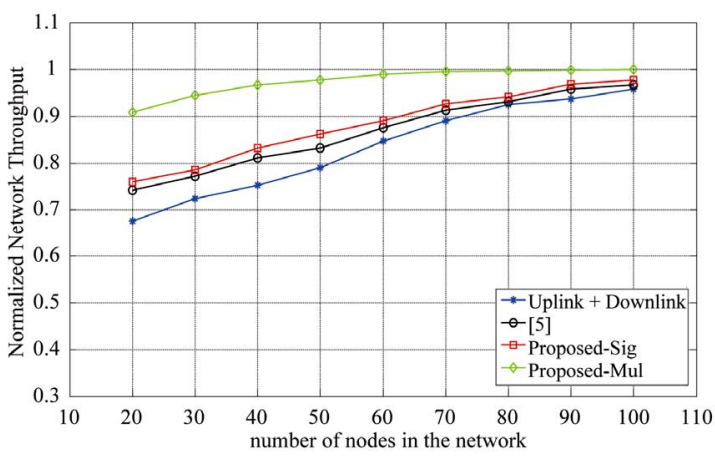

(b)

Figure 8. Normalized network throughput. Service token of all nodes $\in[0,5]$; (b) Service token of all nodes $=\mathbf{1}$. 
Figure 8 shows the normalized network throughput. The figure represents that the proposed algorithm with using multi-channel system is higher throughput than single channel and the proposed algorithm is better than CSLA algorithm which is discussed in [5]. The uplink plus downlink is the worst performance among the schemes because this scheme deprives of exploiting combined uplink and downlink transmissions and so the spatial reuse is lower than other schemes. As expected in Figure 6, Figure 7 and Figure 8, ST does not influence on the CUR, LCR and normalized network throughput so much, because ST cannot independently influence on these metrics.

\section{Conclusions}

An efficient routing tree algorithm is proposed for combined uplink and downlink slot allocation on the centralized scheduling scheme in IEEE 802.16 based wireless mesh networks. This algorithm considers interferences, downlink packets, uplink packets and node identifier for determining parent node in the routing tree construction. This algorithm can reduce interference among the nodes and improve spatial reuse in the network. Also, some related issues such as fairness, relay model, slot reuse and moderating switching frequently between transmitting and receiving in the successive time slots have been considered. This work is compared with combined uplink and downlink slot allocation (CSLA) that is proposed in [5]. Results confirm that the proposed algorithm can significantly increase the link concurrency ratio, network throughput and channel utilization ratio as well as reducing the scheduling length. In conclusion, the presented algorithm will provide a simple routing algorithm for combined uplink and downlink slot allocation on the centralized scheduling in IEEE 802.16 mesh mode.

\section{References}

[1] I. F. Akyildiz, X. Wang and W. Wang, "Wireless Mesh Networks: A Survey," Elsevier Journal of Computer Networks, Vol. 47, No. 4, March 2005, pp. 445-487.
[2] IEEE Standard 802.16-2004, "Local and Metropolitan Area Networks-Part 16: Air Interface for Fixed Broadband Wireless Access Systems," October 2004.

[3] B. Han, F. P. Tso, L. Lin and W. Jia, "Performance Evaluation of Scheduling in IEEE 802.16 Based Wireless Mesh Networks," Proceedings of International Conference on Mobile Adhoc and Sensor Systems, Vancouver, 9-12 October 2006, pp. 789-794. doi:10.1109/MOBHOC.2006.278652

[4] B. Han, W. J. Jia and L. D. Lin, "Performance Evaluation of Scheduling in IEEE 802.16 Based Wireless Mesh Networks," Elsevier Journal of Computer Communications, Vol. 30, No. 4, February 2007, pp. 782-792.

[5] S. Liu, S. Feng, W. Ye and H. Zhuang, "Slot Allocation Algorithms in Centralized Scheduling Scheme for IEEE 802.16 Based Wireless Mesh Networks," Elsevier Journal of Computer Communications, Vol. 32, No. 5, March 2009, pp. 943-953.

[6] Y. Tang, Y. Yao and J. Yu, "A Novel Joint Centralized Scheduling and Channel Assignment Scheme for IEEE 802.16 Mesh Networks," Proceedings of 4th International Conference on Computer Science \& Education, Nanning, 25-28 July 2009, pp. 289-293.

[7] P. Du, W. Jia, L. Huang and W. Lu, "Centralized Scheduling and Channel Assignment in Multi-Channel SingleTransceiver WiMax Mesh Network," Proceedings of IEEE Wireless Com- munications and Networking Conference, Hong Kong, 11-15 March 2007, pp. 1734-1739.

[8] P. R. Sheu, C. F. Hu, C. C. Liou, F. C. Chuang and Y. C. Chen, "An Efficient and Interference-Aware Centralized Routing Tree Algorithm for the Routing and Packet Scheduling Problem in IEEE 802.16 Mesh Networks," Proceedings of International Conference on Communication and Mobile Computing, Shenzhen, 12-14 April 2010, pp. 496-503. doi:10.1109/CMC.2010.340

[9] A. Al-Hemyari, C. K. Ng, N. K. Noordin, A. Ismail and S. Khatun, "Cross Layer Design in 802.16d," Australian Journal of Basic and Applied Sciences, Vol. 3, No. 3, 2009, pp. 1591-1600.

[10] A. Al-Hemyari, C. K. Ng, N. K. Noordin, A. Ismail and S. Khatun, "Constructing Routing Tree for Centralized Scheduling Using Multi-Channel Single Transceiver System in 802.16 Mesh Mode," Proceedings of IEEE Internatinal Conference on RF and Microwave, Kuala Lumpur, 2-4 December 2008, pp. 192-196. 